\title{
IMAGING OF FLUOROPHORES IN CHROMATOGRAPHIC BEADS, RECONSTRUCTION OF RADIAL DENSITY DISTRIBUTIONS AND CHARACTERISATION OF PROTEIN UPTAKING PROCESSES
}

\author{
BERnd StANisLAWSKI ${ }^{1}$, ELODIE SCHMIT ${ }^{2}$ AND JOACHIM OHSER ${ }^{3}$ \\ ${ }^{1}$ Merck KGaA, Laboratory of Bead Characterisation, Life Science \& Analysis, Research \& Development, \\ Frankfurter Straße 250, D-64293 Darmstadt, Germany; ${ }^{2}$ Ecole Européenne Chimie Polyméres et Matériaux, 25 \\ rue Bequerel, F-67087 Strasbourg Cedex, France; ${ }^{3}$ Hochschule Darmstadt, Fachbereich Mathematik und \\ Naturwissenschaften, Schöfferstraße 3, D-64295 Darmstadt, Germany \\ e-mail: bernd.stanislawski@merck.de, elodie_schmitt67@hotmail.fr, jo@h-da.de \\ (Accepted August 8, 2010)
}

\begin{abstract}
A new adjustment calculus is presented to determine the true intraparticle distribution of bound protein within chromatographic beads from confocal fluorescence slice series. The calculus does not require knowledge about optical properties of different chromatographic materials like refractive index and turbidity, but it depends on a parameter which can be adjusted interactively. The algorithm is of complexity $\mathscr{O}(n)$ where $n$ is the pixel number. From the reconstructed data we compute the parameters of the protein uptaking process using a model-based approach. It is demonstrated that the protein uptaking rates of the beads strongly dependent on the conditions of the fluid phase influencing the strength of protein surface interaction.
\end{abstract}

Keywords: bead characterisation, chromatography, confocal laser scanning microscopy, image reconstruction, light attenuation.

\section{INTRODUCTION}

Confocal laser scanning microscopy (CLSM) using fluorophore-labelled proteins is a powerful tool for in situ investigation of mass transfer mechanisms within chromatographic materials. CLSM provides the visualisation of an intraparticle protein distribution (i.e., an inhomogeneous protein density) without mechanical destruction of the resin particle and explanation of absorption and diffusion mechanisms inside many chromatographic absorbents. There is a reach literature on CLSMbased chromatography, see, e.g., Ljunglöf and Hjorth (1996); Ljunglöf and Thömmes (1998); Linden et al. (1999); Dziennik et al. (2003); Hubbuch et al. (2003); Kasche et al. (2003); Harinarayan et al. (2006); Kavoosi et al. (2007); Langford et al. (2007); Ljunglöf et al. (2007); Susanto et al. (2007).

The quantitative evaluation of confocal data, however, has always been elaborate due to typical artefacts of CLSM imaging. One major difficulty in the evaluation of confocal data is the elimination of light attenuation effects. During the experiment, the excitation light and the emitted fluorescence signal are attenuated dependent on the light path through the absorbent matrix. Intensity values, which are detected on the cross-section of a spherical absorbent particle, are disturbed by uneven degrees of attenuation resulting in apparently lower intensity values in optically deeper slices of the CLSM images. Furthermore, the degree of light attenuation depends on the excitation and emission wavelength as well as on type and structure of the absorbent matrix. Several efforts were taken to develop correction methods, see e.g., Heinemann et al. (2002; 2004), and Bankston (2008), where these artefacts are eliminated from experimental data before further processing. Bankston (2008) suggested the use of refractive index-based optical microscopy in order to observe mass transfer in spherical absorbent particles, but this technique seems not to be applicable for a quantitative description of diffusion processes in the particles.

The reconstruction of 3D-data from images obtained by CLSM by compensation of the light attenuation is an image formation procedure which is in some sense similar to the inverse Radon transform applied in tomography. In our setting "reconstruction" has the following meaning: The true density of the fluorophores in a xy-plane perpendicular to the optical axis and of distance $z$ from the microscopic lense is estimated using the data of the set of CLSM-slices which are closer to the lense. For a sound description of a robust incremental compensation of the light attenuation in 3D fluorescence microscopy applied to cellular structures is given by Kervrann et al. (2004). In Susanto et al. (2006a) the light attenuation effects in CLSM data of spherical beads are corrected in two steps. First, one assumes plane wave fronts 
perpendicular to the optical axis, and starting from the Lambert-Beer law, an updating formula for varying protein density is used basing on numerical integration over the just computed values of this density. This means that a correction factor is computed which depends on the absorption of the light along its path through the particle. In a second step the effect of the conical shape of the extinction path between the objectice lens and the focal point is corrected using knowledge about the particle shape and the rotatory symmetry of the protein density. Susanto follows the ideas of Visser et al. (1991) and Margadant et al. (1996), but he uses a simplified model allowing a fast correction basing on integration of the density along lines orthogonal to the optical axis (correction along the x-axis, first suggested in Heinemann et al., 2002).

In the present article we follow the approach of Kervrann et al. (2004) and apply a gradient-based image correction. We start from the differential equation for the unknown light intensity describing the absorption along the path of the incoming and reflected light. In the simplest case of light attenuation parallel to the optical axis, the intensity is subject to a 1st order ordinary differential equation which can easily be solved by Euler's method. This reconstruction depends on an unknown parameter which must be adjusted carefully. Because of the low computation time for the reconstruction of the complete data set, it is possible to adjust the parameter interactively where the result of reconstruction is evaluated subjectively. It turns out that (unter the specific optical conditions and for the specific application) it is sufficient to correct the light absorption parallel to the optical axis, where the conical shape of the extinction path, scattering effects, and refraction can be ignored.

From the experimental data obtained by reconstruction using the CLSM images we compute (i.e., statistically estimate) parameters describing protein uptaking processes where we distinguish between two phenomena. Following Kavoosi et al. (2007), the first one is related to reversible binding of the proteins at the inner surface of the porous beads. Then the concentration profiles of the proteins are subject to the diffusion equation fulfilling certain initial and boundary conditions. The corresponding problem can be solved analytically using Fourier's method. Finally, the unknown diffusion coefficient can be determined by adapting the theoretical concentration profile to the experimental data.

Irreversible binding means that the proteins are fixed in their positions after first contact with the inner surface of the beads, see Susanto et al. (2006b). As a consequence of this phenomenom one observes a sharp step in the concentration profiles, also observed by Langford et al. (2007). This step can be considered as a boundary between two phases, one of high protein concentration and the other one of an extremely low concentration. In this case the protein uptaking can be described as a motion of the phase boundary. We present a mathematical model describing the motion of this boundary. Again, the model parameter (in this case the initial speed of motion) is determined by adapting the model to experimental data.

\section{MATERIALS AND IMAGING}

In this study, the Fractogel ${ }^{\circledR} \mathrm{EMD} \mathrm{SO}_{3}^{-}$(M) strong cation exchanger (Merck Darmstadt, Germany) was used as chromatographic material. As protein material a monoclonal antibody was labeled using ALEXA ${ }^{\circledR}$ Fluor 546, (Molecular Probes, Eugene Oregon, USA) in a low dye to protein molar ratio of 0.7 .

A microfluidic T-switch can be used in order to pipe a switchable loading stream onto chromatographic beads. The 10 to 15 beads were retained in a Teflon tube (Tefzel tube OD 1/1", ID 0.040 " of Upchurch Scientific ${ }^{\circledR}$, which was connected to an outlet tube of narrow inner diameter (Tefzel tube OD 1/16", ID 0.020 " by one-piece Fingertight Fittings, F120, and low pressure union, P 702, all by Upchurch Scientific $\left.{ }^{\circledR}\right)$. At the inlet side the tube was connected via a T-shaped connector (Thru-Hole, P652, Tefzel tube OD 1/16", ID 0.040", connected by one-piece Fingertight Fittings, F120 all by Upchurch Scientific $\left.{ }^{\circledR}\right)$ with two computer controlled syringe pumps $\left(\right.$ Cavro $^{\circledR}$ XLP 6000 Modular Syringe pump equipped by Cavro ${ }^{\circledR}$ Basic Integration Kit). These two syringe pumps were activated alternatively to pump load streams through the tube with a rate of $5 \mu \mathrm{l} / \mathrm{min}$. In this experimental setup the chromatographic beads were in intimate contact with alternating streams of aqueous buffer solution or of buffered protein solution $(10 \mathrm{mg}$ monoclonal antibody/ml). In such a microfluidic setup, the mixing between the load streams is negligible.

During the investigation, it was found that the more simpler method gives identical experimental results.

The chromatographic samples were incubated within $25 \mathrm{mM}$ phosphate buffers at $\mathrm{pH}=6.0$ or in $25 \mathrm{mM}$ phosphate/ $25 \mathrm{mM}$ acetate buffers at $\mathrm{pH}=5.0$. About $5 \mu$ l of chromatographic material was incubated in $1 \mathrm{ml}$ buffer containing $5 \mathrm{mg}$ labeled protein. The samples were kept at room temperature on rollermixer for times of $1,2,4,8,16,32,64$ and 128 minutes. The absorption reactions were stopped by centrifugation. The sedimented particles were washed 
and were applied to microscope slide for immediate CLSM measurement.

Several particles were pipetted out and scanned under Leica CLSM TCS SL using oil immersion objective $(40 \times$ magnification). Fluorophore-labelled protein was excited by $\mathrm{HeNe}$ Laser at $543 \mathrm{~nm}$ and the emitted fluorescence intensity was detected using LP650 filter. The Fractogel ${ }^{\circledR} \mathrm{EMD} \mathrm{SO}_{3}$ particles were scanned in xy-slices using a $1024 \times 1024$ pixel camera, and the distance between neighboring slices was $200 \mathrm{~nm}$. Thus, the pixel size was approximately $0.1 \times$ $0.1 \times 0.2 \mu \mathrm{m}^{3}$, and series of z-scans of a typical particle with a diameter of $80 \mu \mathrm{m}$ consists of about 400 scans. When coding the pixel values with 1 Byte, the content of the corresponding 3D-data can reach 0.5 GByte.

\section{RECONSTRUCTION OF THE SPATIAL DENSITY}

Confocal laser scanning microscopy has been proven very useful for imaging inhomogeneous densities of fluorophores, see Susanto et al. (2006a) and references therein. However, imaging of spatial density distributions involves absorption effects, since radiation traveling in matter is more or less absorbed, i.e., converted into different energy forms, especially heat. This leads to a distortion of the true density distribution of the fluorophores inside the beads. Thus, estimating the radial density distribution supposes a reconstruction of the true density from the image data.
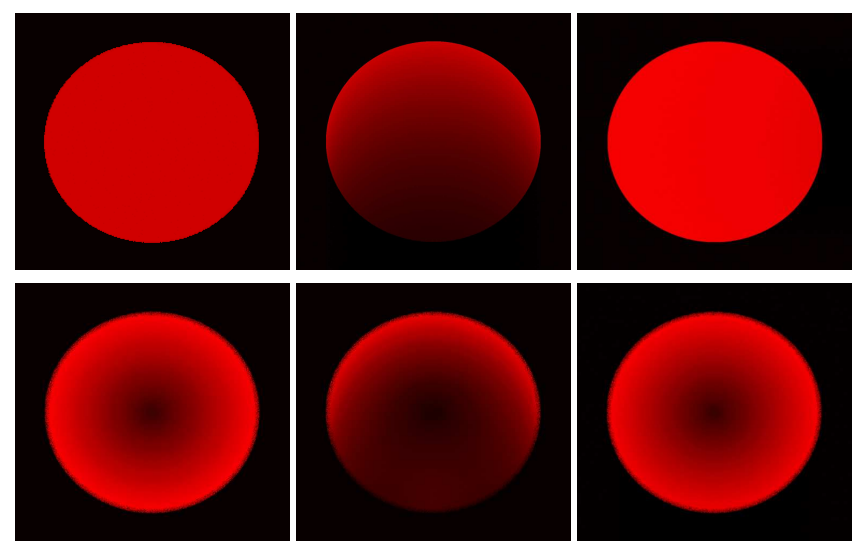

Fig. 1. Demonstration of the reconstruction of the spatial density distribution in a ball. These images show xz-slices of simulated $3 D$ data through the ball's centre. top: homogeneous distribution, bottom: inhomogeneous distribution, left: original data, middle: simulated absorption, right: reconstructed data.

For simplicity we consider the absorption along a straight line parallel to the optical axis of the microscope. That is, the emission path of the light is the reverse of the excitation path. Let $\mathscr{I}(x, y, z)$ and $\mathscr{K}(x, y, z)$ denote the radiant intensity and the mass density of the fluorophores, respectively, depending on the spatial coordinates $x, y$ and $z$. For fixed $x$ and $y$ we use the settings $I(z)=\mathscr{I}(x, y, z)$ and $\kappa(z)=\mathscr{K}(x, y, z)$. Let $I(z) d z$ denote the local radiant intensity in a thin layer of infinitesimal thickness $d z$. It is well known that the change of the intensity is proportional to the intensity itself,

$$
\frac{\mathrm{d}}{\mathrm{d} z} I(z)=-\lambda \kappa(z) I(z),
$$

see, e.g., Section 6.4 .4 in Jähne (2005). Here $\lambda$ is the homogeneous absorption coefficient, $\lambda>0$. More precisely, the product $\lambda \cdot I(z)$ is the local contribution of a mass unit of the fluorophores to the absorption.

The relationship (Eq. 1) is an ordinary differential equation having the solution

$$
I(z)=I(0) \mathrm{e}^{-\lambda \int_{0}^{z} \kappa(t) \mathrm{d} t}, \quad z \geq 0,
$$

where $I(0)$ is the known initial intensity. In the case of a homogeneous density, $\kappa(z)=\kappa$, the solution (Eq. 2) simplifies to $I(z)=I(0) \mathrm{e}^{-\lambda \kappa z}, z \geq 0$. This means that one observes an exponential decay of the radiant intensity. This exponential decay of the intensity in an homogeneous medium is known as the LambertBeer's law or Bourgers's law. In the case of an inhomogeneous medium, however, one can not expect an exponential decay.
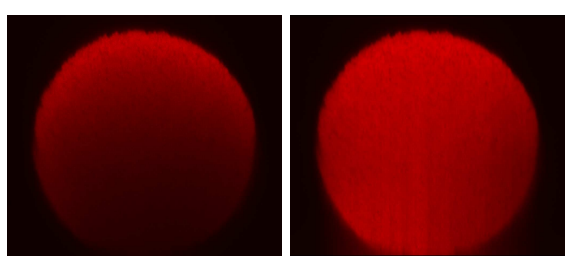

Fig. 2. Reconstruction of the fluorophore density of a CLSM image of a bead. Both images show xz-slices of the 3D data through the centre of the bead. left: original CLSM image, right: reconstructed density.

The analytical solution (Eq. 2) is not very useful for estimating the true density $\kappa(z)$ from image data. First we note that the data are given on grid points, i.e., the input signal is not continuous. Moreover, the problem does not consist in the estimation of an unknown intensity from a given density profile but we are interested in the reconstruction of the density profile from the CLSM signal. 

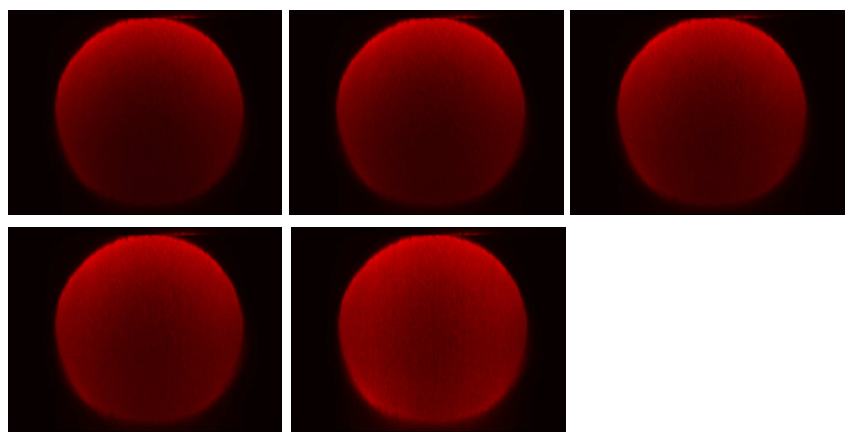

Fig. 3. Reconstructions of the fluorophore density of a CLSM image of a bead for increasing ratio $\lambda / \beta=$ 50, 75, 100, 150, 200 (in lexicographic order). The bead radius is $34.5 \mu \mathrm{m}$ and the incubation time was 3 min. All images show xz-slices of the corresponding $3 D$ data through the centre of the bead.

In our approach, the signal $f$ assigned to a column of a cannel of the CLSM image is modeled as the product $f(z)=\beta \cdot I(z) \cdot \kappa(z)$, where $\beta$ is an unknown coefficient, see Visscher et al. (1994) for the factorisation. The value of $\beta$ is proportional to the absorption coefficient $\lambda$ and it depends on the technical data of the microscope, especially on the properties of the used video camera describing the transition of the intensity of the reflected light into pixel values. However, $\beta$ is assumed to be independent of the intensity $I$. This assumption is important for the reconstruction method presented in the following.

A discretisation of the left-hand side of Eq. 1 gives

$$
\frac{I(z+\Delta)-I(z)}{\Delta}=-\lambda \kappa(z) I(z)
$$

with the pixel size $\Delta$ in $z$-direction. This yields

$$
I(z+\Delta)=I(z)-\frac{\lambda}{\beta} f(z) \Delta
$$

as the basis of the following algorithm for computing the unknown density $\kappa(z)$ at the discrete positions $z_{k}=k \Delta, k=0, \ldots, n-1$ :

1. Compute the intensity $I$ from the recursive formula

$$
I\left(z_{k+1}\right)=I\left(z_{k}\right)-\frac{\lambda}{\beta} f\left(z_{k}\right) \Delta, \quad k=0, \ldots, n-1
$$

using the initial setting $I(0)=1$.

2. Compute the unknown density $\kappa(z)$ from

$$
\kappa\left(z_{k}\right)=\frac{f\left(z_{k}\right)}{\beta I\left(z_{k}\right)}, \quad k=0, \ldots, n-1
$$

for $I\left(z_{k}\right)>0$.
The algorithm is of complexity $\mathscr{O}(n)$, i.e., the computation time is linear in the pixel number $n$. A difficulty of the above algorithm is that the coefficients $\lambda$ and $\beta$ and thus the ratio $\lambda / \beta$ are unknown. In principle, the ratio $\lambda / \beta$ can be determined from the image data exploiting knowledge about symmetry properties, e.g., using the fact that the beads are of spherical shape and the density $\kappa$ is rotatory symmetric about the bead centre. Nevertheless, exploiting symmetry properties has the clear disadvantage that result becomes instable with respect to deviations of the image data from the symmetry assumptions. Therefore, the ratio $\lambda / \beta$ is used as a parameter of the algorithm to be adjusted empirically.

Eq. 5 suggests that $\lambda$ appears as a further parameter of the algorithm to be adjusted. However, when computing a normalised density, the knowledge of $\lambda$ is not necessary.

In the following we demonstrate how the reconstruction method described above works. In order to prove the correctness of the reconstruction we consider simulated data (see Fig. 1). It turns out that the reconstruction are - up to multiplicative constants - the same as the originals. Fig. 2 shows the reconstruction for real CLSM data. Notice that neither in $\mathrm{xy}$-slices nor in $\mathrm{xz}$-slices of the original data it can be seen that the distribution of the fluorophores inside the bead is homogeneous. However, the homogeneity of the distribution can be seen after reconstruction. Figs. 4 and 5 show the reconstruction for CLSM data of beads with inhomogeneous.

Notice that the algorithm presented above is independent of the particle shape and the rotatory symmetry of the protein density. It can also be applied in cases of irregularly shaped particles. Nevertheless, the joice of the ratio $\lambda / \beta$ depends on the image content. An effective joice needs specific properties of the image (e.g., rotatory symmetry of an imaged particle or macroscopic homogeneity of a microstructure).

\section{RESULTS OF RECONSTRUCTION}

In order to describe the main phenomena of protein uptaking we consider two extremal situations:

Reversible binding. In the first case we assume temporary binding of the proteins at the internal surface of the bead. This means that after touching the internal surface, the protein position is fixed for short time, only. Then the process of protein uptaking is dominated by Brownian motion of the proteins 
inside the pore space of the beads and the process can be modeled by diffusion where the time-depending protein concentration is subject to a diffusion equation. See Fig. 4 for an example.

Irreversible binding. Irreversible binding means that after touching the surface, the protein position is fixed permanently. As a consequence, the surface will completely be occupied by proteins which reduces the effective pore space available for further uptaking. One can observe a sharp step in the concentration profile forming a phase boundary. The region of high concentration can be described by a growing spherical shell, i.e., the volume between two concentric spheres of radii $R$ and $r$, where $R$ is the bead radius and $r$ is the radius of a spherical surface inside the bead (forming the phase boundary). Growing of the spherical shell means that the radius $r$ of the internal sphere decreases with increasing processing time $t$, see Fig. 5. The concentration inside the inner ball is much smaller than in the shell.
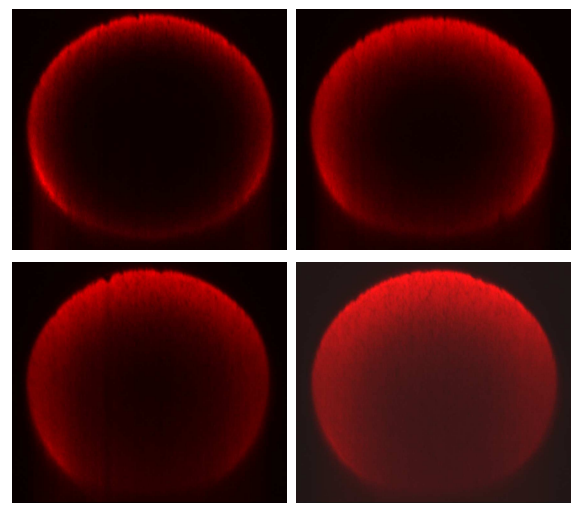

Fig. 4. Reconstructed density profiles from CLSM images, xz-slices, a series of beads uptaken with increasing processing time $t=1 \mathrm{~min}, t=2 \mathrm{~min}, t=$ $4 \mathrm{~min}$, and $t=8 \mathrm{~min}$ (in lexicographic order). The radii of the beads are $30.9 \mu \mathrm{m}, 34.6 \mu \mathrm{m}, 33.3 \mu \mathrm{m}$, and $38.9 \mu \mathrm{m}$, respectively, reversible binding.
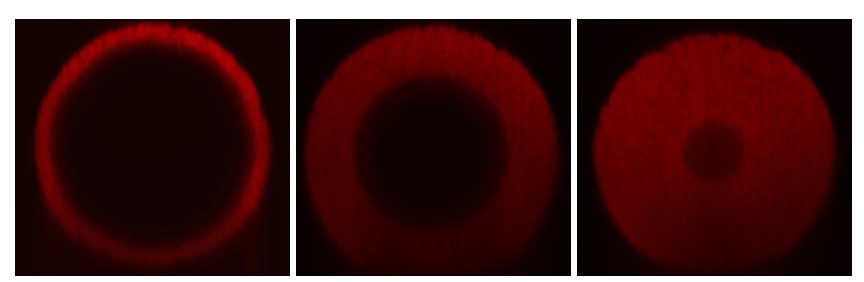

Fig. 5. Reconstructed density profiles from CLSM images of spherical beads (xz-slices) with irreversible binding for increasing incubation time $t=4 \mathrm{~min}, t=$ $32 \mathrm{~min}$, and $t=128 \mathrm{~min}$, respectively.

\section{REVERSIBLE BINDING}

First we formulate the diffusion problem for spherical beads and solve the corresponding partial differential equation. The evolution of the concentration of a fluorophore in a bead is described by a partial differential equation for the concentration $c$ as a function of three spatial coordinates and time, $c=c(x, y, z, t)$. The concentration fulfils the parabolic equation

$$
\Delta c=\frac{1}{D} \frac{\partial c}{\partial t},
$$

where $\Delta$ is the Laplace operator and $D$ denotes the diffusion coefficient. Introducing spherical polar coordinates and assuming rotational symmetry about the origin, the diffusion equation reduces to

$$
\frac{\partial^{2} c}{\partial \rho^{2}}+\frac{2}{\rho} \frac{\partial c}{\partial \rho}=\frac{1}{D} \frac{\partial c}{\partial t}
$$

where the concentration is seen as a function of the radial coordinate $\rho$ and the time $t, c=c(\rho, t)$. We consider the boundary and initial conditions

$$
\begin{aligned}
c(-R, t)=c(R, t) & =1, & t & \geq 0, \\
c(\rho, 0) & =0, & -R & <\rho<R,
\end{aligned}
$$

respectively. The boundary condition (Eq. 7) is valid, if the concentration decrease outside the particle is negligible. The solution of the partial differential equation (Eq. 6) satisfying the boundary conditions (Eq. 7) as well as the initial condition (Eq. 8) can be obtained by Fourier's method. One gets

$c(\rho, t)=1+\frac{2 R}{\pi \rho} \sum_{k=1}^{\infty} \frac{(-1)^{k}}{k} \exp \left(-\frac{(k \pi)^{2} D t}{R^{2}}\right) \sin \frac{k \pi \rho}{R}$

for $-R<\rho<R$ and $t \geq 0$, see, e.g., Jost (2002).

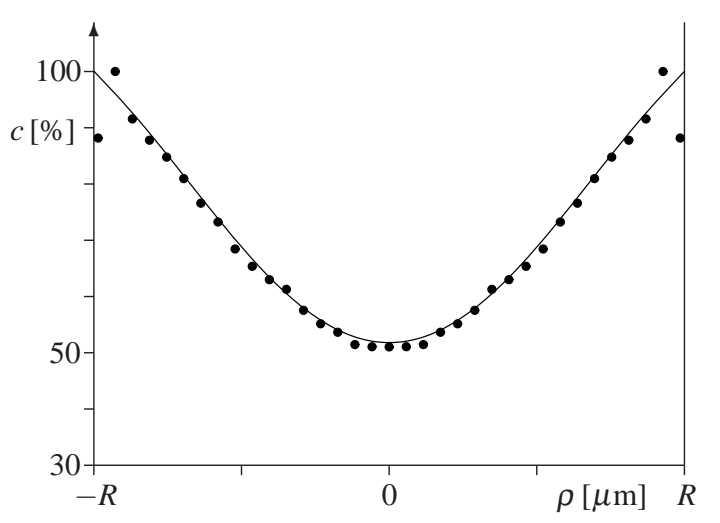

Fig. 6. Experimental data of the concentration profile of the spherical bead shown in Fig. 3 for $\lambda / \beta=$ 
200. The adapted model corresponds to a diffusion coefficient of $D=0.939 \cdot 10^{-12} \mathrm{~m}^{2} / \mathrm{min}$.

Fig. 6 shows the adaption of the diffusion model to the radial density obtained from the CLSM image of Fig. 6 for the optimal correction ratio $\lambda / \beta=200$. Obviously, in this case the protein uptaking is driven by diffusion, only. As a further example we consider a series of beads under different processing times, see Figs. 4 and 7. It turns out that the diffusion coefficient $D$ does not depend on $t$ which means that $D$ is independent of the concentration $c$.

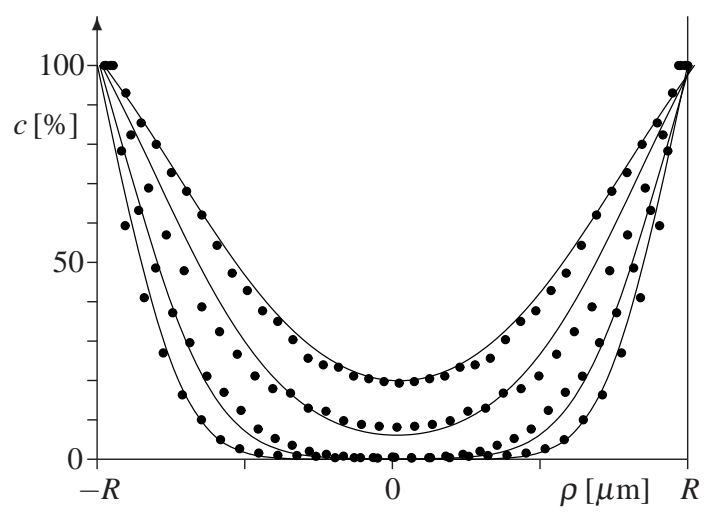

Fig. 7. Experimental data of the concentration profile of the spherical beads shown in Figure 4 for $t=1$, $2,4,8 \mathrm{~min}, t=2 \mathrm{~min}, t=4 \mathrm{~min}$, and $t=8 \mathrm{~min}$. The adapted model corresponds to a diffusion coefficient of $D=0.263 \cdot 10^{-12} \mathrm{~m}^{2} / \mathrm{min}$.

\section{IRREVERSIBLE BINDING}

Clearly, inside the spherical shell the protein uptaking is driven by diffusion, too. Because of the reduced pore space the diffusion is much smaller than in the case of reversible binding. Thus, from the mathematical point of view, irreversible binding can be modelled by the diffusion equation (Eq. 6) but with time varying boundary conditions. Let $p$ denote the fraction of proteins in the spherical shell bounded at the inner surface of the bead, $0<p<1$. Then the boundary and initial conditions (Eqs. 7 and 8) are replaced with $c(R, t)=1$ and $c(r, t)=p$ for $t>0$ and $c(\rho, 0)=p$ for $0<r<\rho<R$, respectively, where the time dependent radius $r=r(t)$ with $r(0)=R$ is subject to the ordinary differential equation

$$
-\left.D \frac{\partial c}{\partial \rho}\right|_{r}=-p \frac{\mathrm{d} r}{\mathrm{~d} t}
$$

i.e., the total flow through the inner surface is proportional to the change of the volume of the spherical shell. See Fig. 8 for a hypothetical concentration profile. The unknown fraction $p$ depends on the structure of the pore space of the bead. We assume that $p$ is close to one. Notice that when the variable inner radius $r$ drops 0 , the protein uptakung process only follows the diffusion law (Eq. 6) with the boundary condition $c(R, t)=1$.

In particular because of the unknown fraction $p$, the above problem is hard to solve. Thus we consider a simplified uptaking model where the growing of the spherical shell is proportional to the ratio of the internal surface area $4 \pi r^{2}$ and the external surface area $4 \pi R^{2}$. It follows that the inner radius $r$ of the shell as a function of the time $t$ satisfies the ordinary differential equation

$$
\frac{\mathrm{d} r}{\mathrm{~d} t}=-v_{0} \frac{r^{2}}{R^{2}},
$$

with the initial condition $r(0)=R$. The solution is

$$
r(t)=\frac{R^{2}}{v_{0} t+R}, \quad t \geq 0 .
$$

Here $v_{0}$ is a model parameter controlling the growing of the shell. It can be interpreted as a speed characterising the velocity of the proteins inside the shell. Small $v_{0}$ corresponds to a slow growing (a low decrease of $r$ ) and, vice-verse, large $v_{0}$ yields a fast growing. From Eq. 9 it follows that the speed $v$ of the shell's growing is

$$
v(t)=\frac{v_{0} R^{2}}{\left(v_{0} t+R\right)^{2}}, \quad t \geq 0 .
$$

It decreases extremely fast with increasing incubation time $t$. Finally, we remark that the parameter $v_{0}$ is the initial speed, $v_{0}=v(0)$.

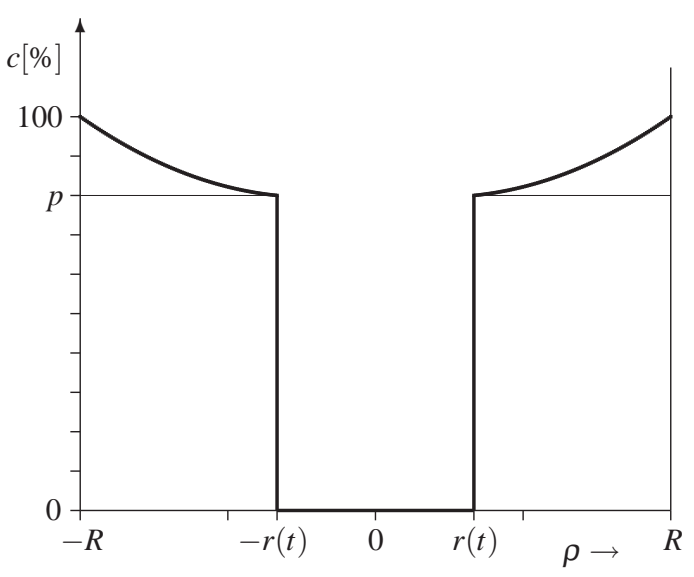

Fig. 8. Sketch of the radial concentration profile of a spherical bead with irreversible binding.

As an example we consider a series of spherical beads for increasing incubation time. Fig. 5 shows a part of this series for incubation times $t=4 \mathrm{~min}$, $t=32 \mathrm{~min}$, and $t=128 \mathrm{~min}$, respectively. Fig. 9 
shows inner radius $r$ of the shell as a function of the incubation time $t$. The mathematical model given by Eq. 9 is adapted to the measurement values obtained from the complete series, where the parameter $v_{0}$ is determined by least squares method. It turns out that the model fits the experimental data well, if the true bead radius is reduced by the mean roughness depth of the bead surface. Here, we assume a reduced radius of $R^{\prime}=22.5 \mu \mathrm{m}$ which is due to a correspondingly roughness depth of the bead surface.

The disadvantage of the simplified uptaking model is that the parameter $v_{0}$ is not comparable with the diffusion coefficient $D$. In so far, reversible and irreversible and irreversible binding can not be compared quantitatively.

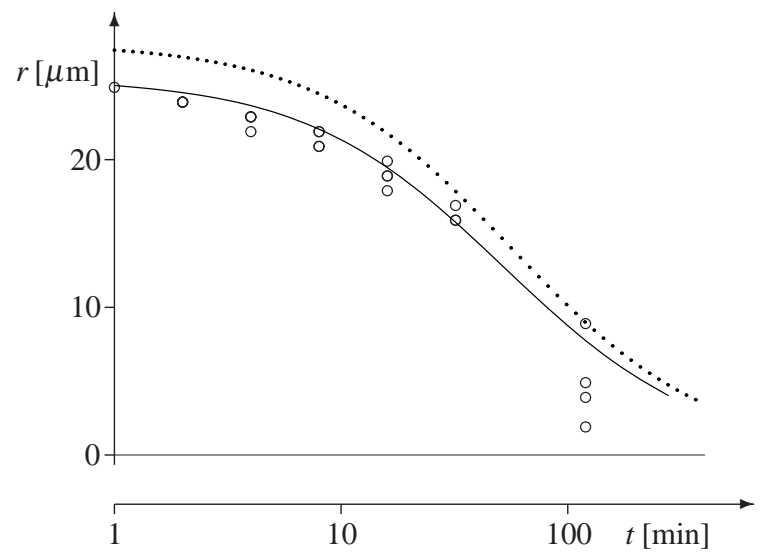

Fig. 9. The inner radius $r$ depending on the time $t$, where the time is in a logarithmic scale. The circles represent the experimental data, the two curves show the function $r(t)$ for the model parameter $v_{0}=8.167$ $\cdot 10^{-9} \mathrm{~m} / \mathrm{s}$; dotted line: the curve for the true bead radius $R=24.9 \mu \mathrm{m}$, solid line: the curve for the reduced radius $R^{\prime}=22.5 \mu \mathrm{m}$.

\section{DISCUSSION}

Industrial protein purification chromatography requires beads having high dynamic capacity. The dynamic depends on the nanostructure of the beads providing a fast diffusion of macromolecules combined with a high concentration of effective protein binding sites. The concentration profiles determined by the method presented in this article show that the same protein can penetrate in completely different ways depending only on a slight change of the $\mathrm{pH}$-value within the aqueous puffer. This conspicuous behaviour has to be explained by constrains of inner structure and electrostatic interaction that affect the mobility of protein molecules within beads.
Insight into the porous nanostructure of beads can be given by scanning electron microscopy (SEM), see Kavoosi et al. (2007); Langford et al. (2007) for microscopic images. In principle, the nanostructure can be seen as a rigid open cell foam consisting of a network of nodes, edges and voids in between. The protein molecules diffuse in the pore space, where the geometric constrains provoked within the open cell foam serves as a geometry factor reducing the rate of diffusion compared to protein mobility in free solution. Fig. 10 shows a mathematical model of the microstructure. The core of the model is a Laguerre tessellation with respect to a dense packing of balls with independent and lognormal distributed diameters $(c v=0.2)$. For details see Ohser and Schladitz (2009), Chapter 7.

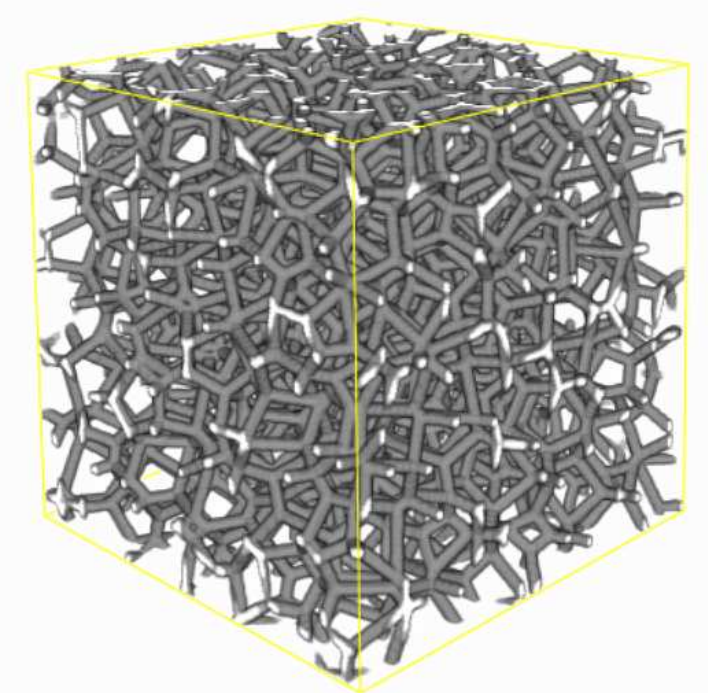

Fig. 10. A mathematical model of the microstructure of the beads. The solid phase is obtained by a dilation of the system of edges in a realisation of a Laguerre tessellation (kindly provided by C. Redenbach).

The primary open cell foam is coated by grafted flexible polymers that carry charged residues. In the investigated chromatographic material, Fractogel ${ }^{\circledR} \mathrm{EMD} \mathrm{SO}_{3}^{-}$, these are negatively charged sulphonate residues $\left(-\mathrm{SO}_{3}^{-}\right)$. In an acidic buffer system the positively charged protein molecules are bound to the sulphonate residues of the polymeric coat by electrostatic interaction. At a $\mathrm{pH}$-value of 6.0 the binding is constant between protein and the anionic polymeric coat is moderate and a major fraction of protein present within the cell can diffuse freely. The diffusion of protein molecules into a bead under the condition of weak electrostatic interaction with the 
polymeric coating the follows an exponential gradient of concentration appropriate to Fick's first law. In contrast, at a $\mathrm{pH}$-value of 5.0 a discontinuous radial profile of bound protein molecules and a much slower mass transfer into the beads has been observed. Apart from the $\mathrm{pH}$-value all experimental conditions have been kept constant. Reducing the $\mathrm{pH}$-value by one unit results into a tenfold higher positive net charge of the protein. Strong cooperative binding to grafted polymers maintains the protein quasi irreversibly at any potential binding site. The exposed pore edges are easily approached by mobile proteins. The pore will be tightened before alternative sites at the cell walls are covered by protein molecules. Additional protein, which enters the cell, protein will be attached to the inner surface of the pore space. Only in those case when the predominant part of the inner surface is completely covered by proteins, advancing molecules can pass the open cell faces in order to cover the inner surface of neighboring cells. The density profile in Fig. 11 gives evidence that irreversibly bounded protein molecules are fixed in their positions while additional molecules diffuse into the pore space until they find free binding sites on the inner surface of the bead. On the nanoscale, in this way the inner surface is occupied cell by cell. Macroscopically, this results in discrete zones which are either protein saturated or protein free. The just tightened cells allows only a considerably lower protein transfer into the interior of the beads.

CLSM provides a direct access to protein density distribution within chromatography beads. The conditions of incubation can be modified over a wide range. The contribution of geometry of the pore space, the inner surface modification and the buffer conditions can be monitored within reasonable time resolution with clear evidence. This gives a better understanding of the uptaking processes and handles for further improvement of chromatographic materials and protein purification conditions. The reconstruction of concentration profiles from CLSM images and the computation of the corresponding model parameters have been proven useful for studying the protein uptaking process.

\section{ACKNOWLEDGEMENT}

The authors thank Mrs. Claudia Wilm for supporting sample preparation and microscopic investigation. Her hints have been very helpful for taking a huge series of high-quality CLSM images.

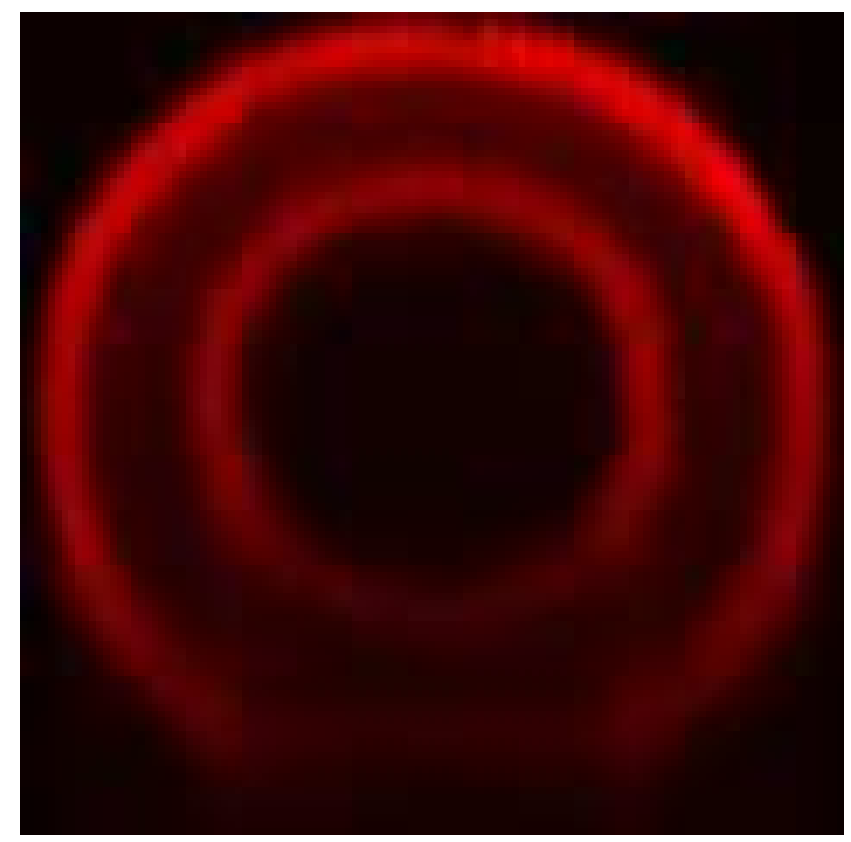

Fig. 11. Density profile of irreversible protein binding in a sequence of labelled protein (2 min), unlabelled protein $(16 \mathrm{~min})$ and labelled protein $(8 \mathrm{~min})$. This figure shows the xz-slice through the bead center showing the density profile reconstructed from a CLSM-image.

\section{REFERENCES}

Bankston TE (2008). Theory and applications of refractive index-based optical microscopy to measure protein mass transfer in spherical adsorbent particles. J Chromatogr A 1188:242-54.

Dziennik SR, Belcher EB, Barker GA, DeBergalis MJ, Fernandez SE (2003). Nondiffusive mechanisms enhance protein uptake rates in ion exchange particles. Proc Natl Acad Sci USA 100:420-5.

Harinarayan C, Mueller J, Ljunglöf A, Fahrner R, Van Alstine JM, van Reis R (2006). An exclusion mechanism in ion exchange chomatography. Biotechnol Bioeng 95:777-87.

Heinemann M, Limper U, Büchs (2004). New insights in the spatially resolved dynamic $\mathrm{pH}$ measurement in macroscopic large absorbent particles by confocal laser scanning microscopy. J Chromatogr A 1024:45-53.

Heinemann M, Wagner T, Douméche B, AnsorgeSchumacher M, Büchs J (2002). Biotechnol Lett A new approach for the spatially resolved qualitative analysis of the protein distribution in hydrogel beads based on confocal laser scanning microscopy. 24:845-50.

Hubbuch J, Linden T, Knieps E, Ljunglöf A, Thömmes J, Kula MR (2003). Mechanism and kinetics of protein transport in chromatographic media studied by confocal laser scanning microscopy, Part I: The 
interplay of sorbent structure and fluid phase conditions. J Chromatogr A 1021:93-104.

Jähne B (2005). Digital Image Processing. Berlin, Heidelberg: Springer.

Jost J (2002). Partial Differential Equations. Berlin, New York: Springer.

Kasche V, de Boer M, Lazo C, Gad M (2003). Direct observation of intraparticle equilibration and the rate-limiting step in adsorption of proteins in chromatographic adsorbents with confocal laser scanning microscopy. J Chromatogr B 790:115-29.

Kavoosi M, Sanaie N, Dismer F, Hubbuch J, Kilburn DG, Haynes CA (2007). A novel two-zone protein uptake model for affinity chromatography and its application to the desription of elution band profiles of proteins fused to a family 9 cellulose binding module affinity tag. $\mathrm{J}$ Chromatogr A 1160:137-49.

Kervrann C, Legland D, Pardini L (2004). Robust incremental compensation of the light attenuation with depth in 3D fluorescence microscopy. J Microsc 214:297-314.

Langford JF, Xu X, Yao Y, Malone SF, Lenhoff AM (2007). Chromatography of proteins on charge-variant ion exchangers and implications for optimizing protein uptake rates. J Chromatogr A 1163:190-202.

Linden T, Ljunglöf A, Kula MR, Thömmes J (1999). Visualizing two-component protein diffusion in porous adsorbents by confocal scanning laser microscopy. Biotechnol Bioeng 65:622-30.

Ljunglöf A, Hjorth R (1996). Confocal microscopy as a tool for studying protein adsorption to chromatographic matrices. J Chromatogr A 743:75-83.

Ljunglöf A, Lacki KM, Mueller J, Harinarayan C, van Reis R, Van Alstine JM (2007). Ion exchange chromatography of antibody fragments. Biotechnol Bioeng 96:515-42.

Ljunglöf A, Thömmes J (1998). Visualising intraparticle protein transport in porous adsorbents by confocal microscopy. J Chromatogr A 813:387-95.

Margadant F, Leemann T, Niederer P (1996). A precise light attenuation correction for confocal scanning microscopy with $\left(n^{4 / 3}\right)$ computation time and $(n)$ memory requirements for $n$ voxels. J Microscopy 182:121-32.

Ohser J, Schladitz K (2009). 3d Images of Materials Structures - Processing and Analysis. Weinheim, Berlin: Wiley VCH.

Susanto A, Herrmann T, Hubbuch J (2006a). Short-cut method for the correction of light attanuation influences in the experimental data obtained from confocal laser scanning microscopy. J Chromatogr A 1136:29-38.

Susanto A, Herrmann T, von Lieres E, Hubbuch J (2007). Investigation of pore diffusion hindrance of monoclonal antibody in hydrophobic interaction chromatography using confocal laser scanning microscopy. J Chromatogr A 1149:178-88.

Susanto A, Wekenborg K, Hubbuch J, Schmidt-Taub H (2006b). Developing a chromatographic column model for bovine serum albumin on strong anion-exchanger Source30Q using data from confocal laser scanning microscopy. J Chromatogr A 1137:63-75.

Visscher K, Brakenhoff GJ, Visser TD (1994). Fluorescence saturation in confocal microscopy. J Microsc 175:1625.

Visser TD, Brakenhoff GJ, Groen FCA (1991). The onepoint fluorescence response in confocal microscopy. Optik 87:39-40. 